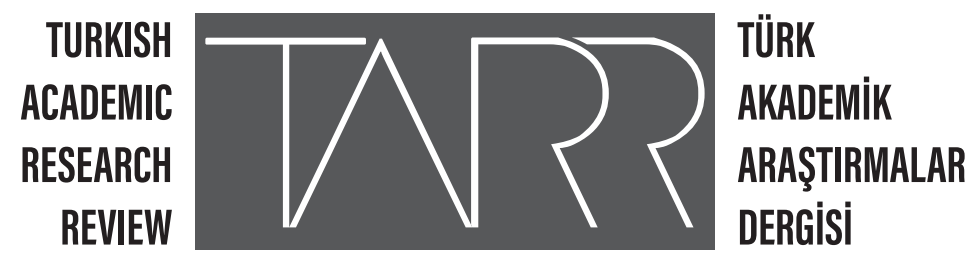

\title{
Modernleşme Sürecinde Müslüman Toplumlarda Karşılaşılan Çağdaş Problemler Üzerine Düşünceler
}

Réflexions Sur Les Problèmes Contemporains Rencontrés Dans Les Sociétés Musulmanes en Cours de Modernisation

\author{
Neslihan ER \\ Dr. DİB Üsküdar Müftülüğ̈̈/ \\ Dr., Religious Afairs Directorious Uskudar Mufti Office. \\ İstanbul / Turkey \\ nesli_er@hotmail.com \\ Orcid 0000-0003-3434-5253
}

\author{
Makale Bilgisi | Article Information \\ Makale Türü-Article Type | Araştırma Makalesi/Research Article \\ Geliş Tarihi-Date Received | 13 Mart/March 2019 \\ Kabul Tarihi-Date Accepted | 24 Aralık/ December 2019 \\ Yayın Tarihi-Date Published | 31 Aralık/December 2019 \\ Yayın Sezonu | Ekim - Kasım - Aralı \\ Pub Date Season | October - November - December
}

Atıf/Cite as: Er, Neslihan, Müslüman Toplumlarda Karşılaş1lan Çağdaş Problemler Üzerine Düşünceler / Réflexions Sur Les Problèmes Contemporains Rencontrés Dans Les Sociétés Musulmanes en Cours de Modernisation. tarr: Turkish Academic Research Review, 4 (4), 423-437. doi: 10.30622/ tarr.539334

Íntihal/Plagiarism: Bu makale, en az iki hakem tarafından incelenmiş ve intihal içermediği teyit edilmiştir. / This article has been reviewed by at least two referees and confirmed to include no plagiarism. https://dergipark.org.tr/tr/pub/tarr

Copyright (C) Published by Mehmet ŞAHİN Since 2016- Akdeniz University, Faculty of Theology, Antalya, 07058 Turkey. All rights reserved. 


\title{
Réflexions Sur Les Problèmes Contemporains Rencontrés Dans Les Sociétés Musulmanes en Cours de Modernisation
}

\author{
Dr. Neslihan ER
}

\section{Résumé}

Les idées et les influences culturelles étrangères influençant les croyances, les traditions et les comportements de la Oumma islamique sont partout présentes. Les problèmes fondamentaux des musulmans d'aujourd'hui sont l'occidentalisation, la modernisation et les conflits avec le monde occidental. Les musulmans n'ont pas suivi l'évolution rapide du monde occidental et sont restés sous l'influence des pays européens. Les frontières de la pensée islamique dans le processus de modernisation et la question de son évolution ont fait l'objet de débats récents.

Les musulmans ont voulu que leurs vies coïncident avec la pensée islamique. Cependant, en raison des développements religieux, scientifique, économique et militaire en Occident des dernières années et qui ont façonné les effets du monde actuel, un processus de transformation globale a débuté. Cette situation a rendu les sociétés dépendantes politiquement et économiquement de l'Occident et les a amenées à ressembler à l'Occident en termes de vie et de réflexion. Lorsque l'occidentalisation s'est accompagnée de phénomènes tels que la modernisation et la sécularisation, les intellectuels musulmans ont produit des pensées et des courants intellectuels de tous genres.

Mots-Clés : Oumma, Tradition, Modernisation, Occidentalisation, Islamiser la Modernité

Modernleşme Sürecinde Müslüman Toplumlarda Karşılaşılan Çağdaş Problemler Üzerine Düşünceler

\section{Öz}

İslam ümmetinin inanç, gelenek ve davranışlarına tesir eden fikir ve yabancı kültür etkileri hemen her yerde mevcuttur. Günümüz Müslümanlarının en temel problemleri, Batılılaşma, modernleşme ve Batı dünyası ile çatışmasıdır. Müslümanlar Batı dünyasında yaşanan hızlı gelişmelere ayak uyduramamış ve Avrupa ülkelerinin tesiri altında kalmışlardır. Modernleşme sürecinde İslam düşüncesinin sınırları ve nasıl tekâmül edeceği meselesi son dönem tartışmalarının konusu olmuştur.

Müslümanlar hayatlarının İslam düşüncesiyle örtüşmesini istemişlerdir. Fakat yakın dönemde Batı'da cereyan eden ve tesirleri günümüz dünyasını da şekillendiren dinî, bilimsel, ekonomik ve askerî alanlardaki gelişmeler nedeniyle küresel bir dönüşüm süreci başlamıştır. Bu durum 
toplumları politik ve ekonomik olarak Batı'ya bağımlı hale getirmiş, yaşam ve düşünüş biçimi olarak da Batı gibi olmaya sevk etmiştir. Batılılaşma beraberinde modernleşme ve sekülerleşme gibi olguları da beraberinde getirince, Müslüman entelektüeller çok yönlü düşünce ve fikir akımları üretmişlerdir.

Anahtar Kelimeler : Ümmet, Gelenek, Modernleşme, Batılılaşma, Moderniteyi İslamileştirme

\section{Giriș}

«La Oumma est définie comme un groupe catégorique qui se reconnaît comme membre de la même religion.» Selon cette définition, un musulman vivant dans un village de Turquie et un musulman vivant dans n'importe quelle partie de l'Afrique est de la même Oumma. La même chose est vraie pour les autres religions; chaque religion est une Oumma avec ses membres. La Oumma islamique est également un groupe formés de musulmans.1

La principale caractéristique qui sépare le groupe catégoriel des autres groupes est qu'il ne forme pas une structure socioculturelle dans un ordre hiérarchique. Les membres de ces groupes dont les frontières sont exprimées en chiffres ne se regroupent qu'en deux figures socioculturelles. Ils se rencontreront dans les temples communs. Ils font ensemble la wakfa à Arafat. Les catholiques font un rituel commun au Vatican. Ils visitent les lieux saints communs et deviennent pèlerins.

S'il y a un problème dans un groupe social et que ce(s) problème(s) affecte(nt) le fonctionnement de ce groupe social, il y a en arrière-plan :

- la réserve humaine ;

- $\quad$ son espace ; sa situation géographique

- $\quad$ son temps ; son histoire

- sa situation économique

- sa formation

- sa structure socioculturelle

- sa situation politique

- sa perception de la religion

Bref, il est nécessaire de regarder les facteurs qui constituent le(s) problème(s), comme la réalité humaine. Chaque être humain, chaque société et même l'humanité entière a eu, a et aura des problèmes. Avoir des problèmes est nécessaire pour le changement social. Le principal est que face aux problèmes qui se posent, les hommes doivent prendre position et produire des solutions. Et ceci est possible avec le savoir.

1 Zeki Arslantürk-Niyazi Akyüz, Din Sosyolojisi II, (İstanbul: Çamlıca Yayınları, 2015). 
Le premier problème de la Oumma islamique est le savoir. On demande aux troupes, pourquoi ne vous battez-vous pas ? Ils répondent nous n'avons pas de poudre à canon. Pas besoin de demander plus. Mais demandons. Pourquoi ? Parce que l'engagement envers les autorités, plus précisément ne pas pouvoir continuer la période appelée l'Age des Lumières Islamiques; ne pas mettre et remettre pardessus. Plus précisément, lors du passage de l'Occident aux Lumières, les sociétés Islamiques ne pouvant ajouter sur Ghazzali, Rabbani, Farabi, Ibn Sina et Ibn Rushd ; ils croient continuer le travail en les imitant. De plus ils ont été informés d'Ibn Khaldoun et de la plupart d'autres environ 200 à 300 ans plus tard. Ces derniers sont grands, mais leurs adeptes sont petits. En rétrécissant de plus en plus dans un cercle vicieux la Oumma islamique se tord.

Les divisions après le Prophète continuent encore aujourd'hui. Les questions et réponses sur "Quel Islam ? ", aujourd'hui comme hier, constituent la source des conflits. Chacun se traite selon son affection; ils se tuent quand c'est nécessaire. À partir de ces déterminations, nous voulons arriver à cette conclusion : aujourd'hui, la Oumma islamique est représentée par «les États ou les mini-États (mini-États tribaux) du mandat». Déjà sur la base politique, le problème est présent. Les dirigeants des États islamiques préfèrent être mandat pour pouvoir être à la tête de leurs gouvernements. Chaque Oumma a un protecteur. Qui est le protecteur de la Oumma islamique ? L'Amérique, la Russie ou la Chine ? Est-ce la Turquie, l'Arabie Saoudite ou l'Iran ? Ces derniers sont-ils aussi des marionnettes? Ou combien est leur pouvoir de protectorat?

L'effondrement de l'Empire ottoman était aussi l'effondrement de la Oumma islamique. Nous ne disons pas que l'Empire Ottoman n'a aucun défaut. Car Allah n'est pas cruel et ne punit personne pour rien. "En vérité, Allah ne modifie point l'état d'un peuple, tant que les individus qui le composent ne modifient pas ce qui est en eux-mêmes.”2

Parmi les problèmes de l'administration des États musulmans, il y a une réponse à la question « quel type de gouvernement ? ». On observe que les administrateurs actuels insistent sur la forme d'administration pour les maintenir au pouvoir ou leur donner le pouvoir. Est-ce une continuation de la tradition ? La monarchie ? La démocratie ? Pour être plus précis, face aux phénomènes État national, démocratie et laïcité (les trois jambes du modernisme), ils sont coincés entre traditionnel, modernité et modernisme. Trois tendances se dégagent dans ce contexte:

- Les traditionnalistes

- Ceux qui disent oui à la modernité, non au modernisme

- Les partisans du modernisme

Ce sont les discussions des gestionnaires et des intellectuels. Le peuple vit sa propre vie et ferme souvent ses oreilles aux intellectuels. Cette situation crée le dilemme de peuple-intellectuel; les deux classes assument que leur propre connaissance représente la vie elle-même.

Parmi les problèmes de la Oumma islamique, il y a avec l'ignorance, sa situation géographique c'est une zone où d'autres ont les yeux. Au premier millénaire, le continent Africain a été ex-

2 Sourate Ar-Ra'd, verset 11. 
ploité par la Oumma Chrétienne et de nombreuses communautés ont été christianisées. Les missionnaires chrétiens opèrent en Asie et sur les communautés islamiques problématiques avec tous leurs moyens. Le Judaïsme, le Christianisme et l'Islam ont émergé du Moyen Orient. Les trois Oummas ont aussi des ambitions politiques, économiques et religieuses dans cette région. Elle est toujours dominée par la Oumma Islamique. Huit Croisades ont été organisées par les Occidentaux dans le but de la sortir des mains de la Oumma Islamique et elles ont échoué à chaque fois. La Oumma Chrétienne Occidentale, qui comprend qu'elle ne peut pas capturer la région par la guerre, veut gouverner ou diriger la région en la divisant. Dans ce contexte, ils ont introduit un nouveau projet appelé l'Initiative pour le Grand Moyen-Orient (Great Middle East Project); ont élaboré des politiques visant à réglementer la région dans le sens de leurs propres intérêts et ambitions.

Outre les problèmes de petite échelle des sociétés et des pays qui représentent la Oumma Islamique nommés pays Islamiques, trois problèmes fondamentaux de plus grande échelle peuvent être considérés : «l'incapacité à déterminer leur position face à la modernisation, à la construction de l'État national et au terrorisme».

\section{Projet d'Islamiser la Modernité}

Les idées et les influences de la culture étrangère sur les croyances, les traditions et les comportements de la communauté islamique sont présentes à peu près partout. Les problèmes les plus fondamentaux des musulmans d'aujourd'hui sont l'Occidentalisation, la modernisation et le conflit avec le monde Occidental3.

\section{Entre Traditionalisme-Modernité}

Les musulmans n'ont pas été en mesure de faire face au développement rapide du monde Occidental et sont restés sous l'influence des pays Européens. Les limites de la pensée Islamique dans le processus de modernisation et son évolution ont fait l'objet de récents débats.

Daryush Shayegan note que l'attente dans la société islamique en général, de l'influence occidentale et la modernité basée sur l'occident entraîne des solutions miraculeuses aux inégalités sociales et aux effondrements moraux, provoquent le rejet des temps nouveaux et créent de nombreux environnements de résistance dans le monde actuel.4

La relation entre tradition-modernité et théorie de la modernisation a commencé à être débattue dans les sociétés non occidentales sous-développées. En particulier, les approches qui prétendent expliquer le problème du développement du point de vue sociologique, d'une part, discutent des conditions de conflit entre la modernité et la tradition et, d'autre part, ont pris pour sujet de recherche les obstacles de la tradition au processus de développement.5

3 Neslihan Er, Moderniteyi İslamlaştırma Projesi: Al Adl Wal Ihsanne Cemaati Modeli, (M.Ü. Sosyal Bilimler Enstitüsü, These de Master non publiée, 2014).

4 Daryush Shayegan, Yaralı Bilinç, (İstanbul: Metis Yayınları, 2012), p.11

5 Fahrettin Altun, Modernleşme Kuramı, (İstanbul: Küre Yayınları, 2011), p. 68, 74, 82. 
Les musulmans veulent que leurs vies se recoupent avec la pensée islamique. Mais un processus de transformation mondiale a été engagé à la suite des récents développements dans les domaines religieux, scientifique, économique et militaire qui ont façonné l'Occident et dont les effets ont pris forme dans le monde actuel. Cela a rendu les sociétés politiquement et économiquement dépendantes de l'Occident et les a amenées à ressembler à l'Occident en tant que forme de vie et de pensée. Lorsque l'Occidentalisation a accompagné la modernisation et la sécularisation, les intellectuels Musulmans ont produit des pensées et des idées multidimensionnelles.

Presque tous les mouvements Islamiques contemporains nés dans le monde islamique depuis les années 1970 ont privilégié l'Islam en tant qu'idéologie politique qui couvre l'ensemble de lordre social avec la compréhension politique de la société. En conséquence, la société idéale pour les Musulmans n'est pas seulement une société composée de croyants, mais sa structure et son système doivent également être Islamiques. À partir de là, aucun pays Arabe ne peut être considéré comme Islamique, car cette idéologie rejette les démocraties parlementaires et, en même temps, les régimes militaires. Les monarques sont également inacceptables.

En principe, les Islamistes sont contre la démocratie Occidentale car elle définit que la souveraineté vient du peuple. En fait, pour eux, la souveraineté ne peut venir que d'Allah. D'autre part, selon Bernard Lewis, l'autorité du roi est sévèrement limitée dans l'Islam. Cette limitation vient de la 'charia' elle-même : ce n'est pas l'état qui produit la loi, mais elle est elle-même créée et protégée par cette loi de Dieu. Le devoir du dirigeant est comme tous les citoyens de préserver et de renforcer cette législation à laquelle il est soumis. La charia est un système de jurisprudence qui insiste sur le fait que l'État n'est pas l'autorité unique et freine ainsi l'autoritarisme.

\section{La Laïcité et la Oumma Islamique}

Pendant des siècles, la ligne directrice de l'histoire a indiqué 'la fin du religieux' et la sécularisation de nombreux pays semblait le confirmer. Selon ce schéma, la religion semble avoir été limitée à l'espace privé. Cependant, parallèlement à ce mouvement de laïcisation, la fin du XIX. siècle et le début du XXe siècle a marqué le retour du religieux et la montée de l'Islamisme dans le monde Arabo-Musulman.6

Au fur et à mesure que la laïcité commençait à se répandre, les débats sur le développement contemporain dans le monde islamique ont toujours eu lieu concernant l'adoption de cette voie ou de voies alternatives. Les débats persistants sur les relations entre religion-État et la laïcité dans le monde Islamique concluent d'une part, que l'islam ne domine que comme une croyance privée et de la séparation de la religion et l'état, et d'autre part, comme dans l'exemple de l'Iran postrévolutionnaire, il y a des opinions qui ont fait valoir leur coexistence.

L'appui ouvert à de telles idées a ouvert la voie à l'émergence du mouvement al-Ikhwan al-Muslimin en 1928, qui a accusé à la fois la laïcité et le Azhar (université égyptienne) de ne pas s'opposer à ceux qui prônent ce mouvement. Le leader du mouvement Hassan al-Banna a fait appel à la renaissance de modèles islamiques tels que la réinstauration de la charia et de la

6 Okasha Ben Elmostafa, Les Mouvements Islamiques au Maroc, (Paris: L'Harmattan, 2007), p. 11. 
zakat et a accusé le modèle Occidental de créer des inégalités socioéconomiques. Depuis 1930 en Égypte, il existe une tension entre l'État laïc et les mouvements religieux appelant à un retour collectif islamique. 7

\section{La modernité ou le Modernisme?}

À la source de la modernité occidentale se situe le processus de découvertes géographiques venu à l'ordre du jour dès le XVI. siècle et les politiques de colonialisme qui en ont découlé.8 L'Occident moderne, devenu juge à l'ère des lumières, a importé les intellectuels de ces pays avec les écoles qu'il a ouvertes, tout en important ses propres cultures dotées d'un pouvoir économique dans d'autres pays du monde. En ce qui concerne le monde islamique, fut concerné, au début, dans un sens particulier l'État ottoman et au sens général toute la géographie musulmane.

La confrontation du monde islamique avec l'Occident moderne et ses conséquences continuent. Le monde islamique, en étant exposé à ces effets socioéconomique, politique et théologique, a dû faire face à de nouvelles situations dans son monde spirituel et dans la vie pratique. La nouvelle situation a influencé intensément le clergé et les groupes religieux et ils ont dû développer différents réflexes contre l'Occident contemporain et ses créations.

L'idée de modernisation européenne, en créant un seul type d'homme, en le massifiant dans son propre sens et en le coupant de sa propre tradition, bloquant ainsi l'opposition, et en l'enfermant dans le caractère de consommateur, est devenu synonyme de civiliser.9

Les intellectuels autochtones qui sont devenus une sorte d'ambassadeurs volontaires de l'Occident ont réglé le nationalisme dans les pays islamiques. Ils ont essayé d'influencer la pensée du peuple en faveur de l'Occident. En outre, les minorités non musulmanes étaient cultivées afin d'enraciner l'exploitation Occidentale et dans tous les sens du terme, afin d'influencer intellectuellement les populations en faveur de l'Occident.

La modernisation dans le monde islamique peut être divisée en plusieurs phases. La première phase correspond à la période allant de la fin du XVIIIe siècle au début du XXe siècle, caractérisée par l'effondrement des systèmes d'État musulmans et l'imposition de la supériorité territoriale et commerciale de l'Europe. La deuxième étape est celle où les élites des pays Islamiques tentent de donner une identité politique moderne à leurs sociétés par le biais des États nationaux établis au XXe siècle, favorisant les changements économiques et sociaux. Dans la troisième phase, il y a un débat sur la direction des développements actuels et le rôle ultime de l'Islam.10

On a déclaré que l'idée de liberté était compatible à la justice en religion, que la sainteté du pays était confirmée dans les textes religieux, que l'égalité et la fraternité devaient être prioritaires et qu'il faut être une société de volontés plutôt qu'une société d’obéissance. Les problèmes de

7 Talip Küçükcan, “Modernleşme ve Sekülerleşme Kuramları Bağlamında Din, Toplumsal Değişme ve İslâm Dünyası", İslâm Araştırmaları Dergisi, 13, (İSAM 2005): 109-128.

8 Fahrettin Altun, Modernleşme Kuramı, (İstanbul: Küre Yayınları, 2011), p. 18.

9 Ali Şeriati, Medeniyet ve Modernizm, (İstanbul: Yeni Zamanlar Yayınları, 2003), p. 21-32.

10 Ira M. Lapidus, Modernizme Geçiş Sürecinde İslam Dünyası, (İstanbul: IFAV, 1996), p. 14, 17. 
l'Occident ont été transférés dans le monde islamique et intériorisés. Appelée aliénation culturelle, la raison occidentale a été louée, la religion a été la première à l'approuver et a perdu sa valeur en tant que culture présentée à son approbation.

La modernisation des États musulmans s'est faite selon deux modèles : contre la religion et avec la religion. La première est sans aucun doute le Kémalisme et la seconde est le Réformisme Musulman.11 La modernisation ou les mouvements réformistes du monde islamique font désormais partie de l'interprétation religieuse. Certains oulema de l'Empire ottoman ont soutenu les activités de modernisation.

Lépreuve de force entre le religieux et la modernité a eu une place et une priorité efficaces, en particulier dans la pensée islamique du siècle dernier. Un certain nombre de débats ont/et ont eu lieu parmi les thèmes les plus importants des intellectuels musulmans, depuis la manière dont les relations avec l'Occident seront codées, jusqu'à quels critères détermineront le comportement des musulmans dans le contexte de la modernité.

Le monde musulman qui tente de façonner aujourd'hui la modernité, ne semble pas réussi beaucoup d'étapes en ce qui concerne l'élimination des obstacles théoriques et pratiques à la réévaluation de sa position. 12

L'Islam et l'Occident ne sont plus simplement deux civilisations rivales concurrentes, voire deux modernités différentes. Ils sont maintenant en pleine complexité avec la réalité du monde contemporain qui s'établit ensemble, parfois en combat et parfois en partenariat coopération. L'erreur de l'identification de l'Occident à la modernité ajoute de nouvelles dimensions à la construction du conflit de réalité.13

L'une des idées fausses conceptuelles les plus courantes dans le débat Islamique et Occidental à l’ère moderne a été l'identification de «l'Occident» avec la «modernité». Cette conception erronée a une influence significative sur les tentatives de compréhension de la relation entre l'Islam et l'Occident à l'époque contemporaine, ainsi que sur ceux qui étudient les processus de modernisation dans le monde Islamique.

Ces analyses conduisent à la conclusion que la «Modernité» n’est pas seulement «Occidentale» mais aussi «l'Occident» n'est pas que «modernité», que l'identification de «l'Occident avec la modernité» a des conséquences négatives importantes sur la relation entre l'Islam et l'Occident. La modernité et l'Occident sont deux concepts et domaines d'existence historiques différents. Utiliser ces termes pour se substituer l'un à l'autre signifie inviter une complexité inutile, créant des conflits et des contradictions potentiels.

Les projets de modernité dans les pays occidentaux et Islamiques ont une propriété entièrement séparée. En Occident, l'histoire et la réalité sociale sont devenues le résultat final naturel

11 Ernest Gellner, Müslüman Toplum, (İstanbul Kabalcı Yayınevi, 2012), p. 114.

12 Erdal Baykan, "Modernliği ve Dindarlığı Uyuşum ve Ayrışma Hâsılasının Ötesinde Okumak Mümkün mü?", Dindarlık Olgusu Sempozyum Tebliğ ve Müzakereleri, (İstanbul: Kur'an Araştırmaları Vakfı, 2004), 141143.

13 John Obert Voll, “Batı'nın Modernite ile Özdeşleştirilmesinin Yanlışlı̆̆ı”, Bilimname, 3, (2003), 87-99. 
et, dans d'autres pays, l'adversité de l'acceptation ou du rejet d'un isme du résultat de l'affectation, de l'imitation a été un acte de souffrance. L'occident elle-même nous a directement et indirectement imposé son modernisme.

Le monde colonial islamique a été entrainé ou contraint au modernisme au point d'imposition intellectuelle et d'assurer la continuité de la colonisation. Les musulmans étaient sous l'influence du modernisme et remirent en cause l'adaptation de l'Islam au modernisme, et certains penseurs pensaient que la réconciliation était possible.

En acceptant la modernisation comme un développement universel, l'occident pensait que le modernisme européen était le seul moyen de développer l'humanité. Les attitudes occidentales sont devenues un exemple à suivre pour les objectifs du modernisme dans les pays du tiers monde, mais dans le processus historique le 'modernisme universel' n'est plus apparu comme un concept généralement accepté en raison de différentes réalités et de différences évidentes avec les mesures de l'Occident dans les sociétés en général. Au lieu de cela, l'approche adoptée a été la conviction que l'économie capitaliste mondiale est une source de changement économique, technologique et de classe dans les sociétés non Occidentales et qu'elle n'est ni un modèle Occidental uniforme ni une société moderne. Le modernisme dans le tiers monde est né de l'effort que l'élite indigène a consommée pour la reconstruction de leurs propres sociétés. Parce que les sociétés qui veulent se moderniser ont à profiter de l'accumulation de l'Occident représentant des valeurs universelles et modernes et aller de l'avant.

Outre les traditionnels autochtones, les discussions entre les intellectuels qui appréciaient la pensée critique, la rationalité et la libre enquête ont été relancées grâce aux relations entre le monde occidental et le monde islamique. Les réformistes ont bénéficié de diverses technologies pour promouvoir leur pensée. L'argument principal est que les sociétés Islamiques sont en arrière contre la technologie Occidentale. Dans la seconde moitié du XIX. siècle, il a tenté de s'adapter rapidement à la vitesse de la génération suivante, de s'informer sur les progrès scientifiques et technologiques. De nombreux écrivains Musulmans ont examiné de manière critique la situation générale de leurs sociétés et ont révélé l'incapacité de l'éducation, de la recherche scientifique, de l'équipement, etc. et l'idée d'une régénération Islamique a été développée, influencée par la nécessité d'adapter la société Islamique aux réalités de la vie moderne. Les jeunes formés dans les écoles occidentales ont apporté une contribution significative aux réformes menées (telles que les régimes constitutionnels, les réformes juridiques et sociales). Le modèle de cette nouvelle élite a été le système Occidental (demandes de souveraineté populaire, modernité Occidentale, libertés individuelles, justice sociale, éducation pour tous, libération des femmes...). Les mouvements Islamistes modernistes et innovants visent à relier le monde islamique au modernisme universel.

Ainsi, presque partout dans le monde Islamique sont nés des mouvements réformistes ou des sociétés ouvertes par des intellectuels et des jeunes cadres. Leur objectif était de sensibiliser le public et d'accélérer le dynamisme social et politique afin de créer des institutions compatibles avec les idéaux de la modernité, à savoir de la liberté et de l'égalité. Dans le même temps, les réformateurs ont contribué à la création d'un grand nombre d'universités dans le modèle Oc- 
cidental et à la diffusion des technologies et des idées permettant d'améliorer le niveau de vie des personnes, telles que la presse, la télévision et Internet.

La France et l'Angleterre sont accusées de vouloir dominer le monde Islamique et de nombreux mythes se sont effondrés, notamment la non-destruction du monde islamique, la suprématie de la culture et de la civilisation Islamiques et la non-destruction de la Oumma. En outre, les idées de réforme qui ont du mal à faire face à la tradition autochtone ont perdu toute légitimité. Ils nont pas pu résister aux institutions religieuses traditionnelles et sont refusés par l'Occident.

Bien que la critique du modernisme ait considérablement augmenté aujourd'hui, elle n'a pas perdu de son impact. À tout le moins, les préoccupations modernes des approches fondamentales de la pensée Islamique rendent discutable l'authenticité de la pensée. On pense que l'Islam, en tant que religion émanant de la révélation et nétant pas évalué avec les critères qui lui sont révélateurs, c'est-à-dire le critère de la révélation au centre de la pensée et n’en bénéficiant pas, nuit à «'Islamité» de la pensée islamique. La solution des problèmes consiste au départ à les problématiser, et il est suggéré qu'il ne sera pas possible de problématiser sans une méthodologie originale, qui ne peut être réalisée avec des modèles modernes.

La pensée bipolaire a changé la perception de l'Islam, en adoptant des concepts laïques et nationalistes que l'Islam moderniste ou les futurs pays Islamiques auraient. Les modernistes se sont tourné vers la mise en forme de l'Islam avec la réconciliation des formes étatiques et économiques Européens. L'acceptation de la pensée moderne entraînait l'idée de changement pour s'y adapter.

Le modernisme Islamique, la doctrine des élites politiques musulmanes et des intellectuels au XIXème siècle, se distingue de la réforme Islamique, la doctrine de l'oulema. Dans l'idée de la réforme islamique, qui a été séparée du modernisme islamique, les ulémas ont pris le rôle principal. Le début de la réforme islamique est antérieur au modernisme. Au XVII. et XVIII. siècle, il se posait auparavant du problème de la supériorité de l'Europe.

Bien que la pensée de réforme Islamique s'opposent au colonialisme et à l'élite admirateur de l'Europe, certains des partisans de la réforme de l'Islam se sont tournés aujourd'hui vers la ligne des modernistes Islamiques. Plus précisément, avec l'opposition des points de sortie, les méthodologies contiennent des éléments de l'idéologie moderne. Aujourd'hui, c'est la raison pour laquelle quand nous appelons réformiste ou moderniste, nous ne pouvons pas clairement articuler la différence entre les deux. Comme il existe des penseurs qui sont décrits comme des modernistes, mais avec des caractéristiques réformistes, le contraire aussi est vrai. Mais dans les deux cas, le point commun est que la situation actuelle est inadéquate et que «l'innovation et le changement» sont une priorité constante.14

Cette réinterprétation de la religion islamique ne peut être perçue comme une sorte de réforme de la religion de l'Occident, car les fondements fondamentaux de la religion n'ont subi aucune modification. Seules certaines perceptions erronées de l'histoire sont réglées.

14 Charles J. Adams, "Fazlur Rahman ve Klasik Modernizm", İslam ve Modernizm: Fazlur Rabman Tecrübesi Sempozyum Kitabı, (İstanbul: İ. B. B. Yayınları, 1997), 85. 
Il n'est pas réaliste d'affirmer que le modernisme Islamique, qui se développe par rapport aux mouvements d'animation de la période classique, est formé par l'influence Occidentale. L'idée du modernisme islamique depuis la Renaissance ne considère pas comme développement la conception mondaine, les événements qui ne dépendent pas des valeurs Islamiques. Le modernisme Islamique, visant la liberté, l'innovation, l'approche scientifique considère le Coran comme une solution. Il croit que nos problèmes seront résolus par l'interprétation du Coran d'une manière actualisée.

Le moyen de se débarrasser de cette dépression est de revenir sur la vision dynamique du monde du Coran, de réfléchir et de tirer parti des points de vue Coranique des conclusions scientifiques modernes. Les modernistes tels que Abduh, Iqbal, qui ont reconnu l'Occident de manière critique, lui ont adopté certaines méthodes pour faire revivre la contemplation de l'Islam. Parce que la religion est vécue dans l'unité de la vie, si le succès n'est pas atteint dans les domaines philosophie, art, etc., le succès souhaité dans la vie religieuse ne peut être atteint.15

Ces modernistes pionniers, dans leur oppression d'être sous domination coloniale, voient comme seule cause ultime de ces conditions : "L'Islam est mal interprété et mal compris par la communauté. » Ils ont pensé que l'Islam, avec le temps, s'est éloigné de la pureté du Temps du Prophète, a été envahi par les innovations et les superstitions et qu'il est difficile de ramener l'Islam à sa pureté d'autrefois. Ils ont soutenu que dans les conditions sévères du monde islamique, ils peuvent s'en débarrasser en prenant la raison en préliminaire dans chaque travail, y compris la religion, et l'adaptation de l'Islam aux conditions modernes, en 'réformant' non pas l'Islam, mais la vie Islamique.

Les principes d'Ibn Taymiyyah «retour de l'Islam à l'origine», « retour au temps des prédécesseurs ", «rejet de l'imitation» et «élimination des innovations» ont également affecté les islamistes modernistes. Parce que ces principes sont considérés comme des références fondamentales menant à une compréhension Islamique authentique. Et le rejet de l'imitation éliminera l'autorité, le dogmatisme et la scolastique dans la pensée. Et avec le rejet des innovations, les superstitions entourant l'Islam seront rejetées.16

\section{Islamiser la Modernité}

La modernité engendre les mêmes incompatibilités avec les différents niveaux d'extensions et est entravés par les mêmes obstacles tant qu'un recueil matériel, équipement, style et méthode habituelle ne peuvent être considérés comme auto-proclamée par une société.

La modernité occidentale dans le monde Arabe a transformé les modes de vie et conduit à l'industrialisation, l'urbanisation et la formation de couches sociales. Il a également inclus la pratique de l'État-Nation indépendant de l'environnement religieux. Les mouvements réformistes sous colonial ont accepté la modernité et ont pensé que les sociétés Arabes devaient concilier le modernisme avec leur religion afin de compenser leur retard technologique à l'Occident

15 Recep Eren, “Prof. Dr. Mehmet S. Aydın'a göre Türk Toplumunun Modernleşmesi”, İstikamet, 3, (2011), 1-4, 18-21.

16 Daryush Shayegan, Yaralı Bilinç, (İstanbul: 2012), p. 183. 
tout en protègent leur identité Islamique. Après leur indépendance, les nouveaux États ont remodelé la modernité occidentale. Cependant, les problèmes sociaux et économiques apparus en 1970 ont conduit les Islamistes à s'opposer au modèle Occidental. Ainsi, les mouvements Islamiques établis à cette époque sont devenus le produit de la modernité. «Lémergence des Islamistes est une réaction à la modernité archaïque ou, plus précisément, une tentative pour trouver le moyen de supporter le lourd fardeau de la modernité, qui est de détruire les anciennes structures, sans se substituer à l'une de ses qualités et à celles du groupe ».

Dans ce cas, la question est de savoir si l'objectif des Islamistes est de moderniser l'Islam ou d'Islamiser la modernité.

Des penseurs novateurs tels que Abduh et Afgani ont proposé de moderniser le patrimoine religieux. Il semble que l'héritage soit la «source» et qu'il ne s'agit que de la moderniser. Au lieu de moderniser l'héritage Islamique, l'Islamisation de la modernité était la proposition de la prochaine génération de penseurs et s'est développée spécialement dans des conditions différentes, telles que l'abolition du califat Ottoman. Le prédécesseur de cette deuxième génération peut être appeler Rashid Riza et les Frères Musulmans. Avec eux, la question de la dualité de modernité-héritage est devenue une question d'identité.

Dans les années 1970-1980, les Islamistes ont rejeté le modèle de la modernité élu par les États existants et ont proposé d'Islamiser cette modernité. Les Islamistes, bien sûr, ne sont pas non plus des traditionalistes qui veulent un revirement de la technologie moderne.

Ils ont réconcilié la modernité avec les développements scientifiques, mais ils ont rejeté les transformations sociales provoquées par ces développements, en particulier la liberté des femmes, et ils veulent « Islamiser la modernité ». Le terme «modernité» a été utilisé dans diverses significations. La modernité, à l'instar d'Olivier Roy, est également envisagée en termes de distance à l'individualisation, les identités et adhésions.

Certaines des questions précieuses pour le mouvement salafiyya ont été développées par les Islamistes. Les pressions étrangères et le despotisme des dirigeants (tâghout) est tenu responsable de la régression des musulmans. La modernité, indispensable dans le domaine industriel et technique, est considérée comme une brutalité sur le plan moral : toute l'humanité a régressé à cause de s'être écarté de ses racines spirituelles. La défense de l'islam et l'opposition de la pensée athée-matérialiste ont conduit à la nécessité de modernisation dans la théologie islamique: les revendications des islamistes sur «l'islamisation de la modernité » sont apparues à ce stade. La lutte contre toute forme de religiosité populaire vu comme «superstition»-les Murabitun, les zawiya(s) ou les sectes ...- est un autre problème reconcidéré par la Salafiyya.

Il semble que: le mahdisme, le wahhabisme ou le réformisme salafiyya, tous ces mouvements sont enregistrés dans le contexte de la conscience de l'Islam et de la crise de l'occupation étrangère; leur soucis commun est pourquoi la Oumma a régressé. La méthode qu'ils ont offerte pour compenser cette tension a été un appel à faire revivre la moralité islamique dans le domaine des relations sociales et des traditions; mais ils se sont divisé sur la réexpression des principes sociaux et politiques de l'Islam afin de pouvoir les adapter aux exigences du monde moderne. 
Lémergence des mouvements islamiques semble être une réponse à la modernisation imposée par l'Occident. Les islamistes ont refusé de moderniser l'Islam contre la modernité perçue comme étrangère entre 1970 et 1980 et, au contraire, ils ont proposé l'islamisation de la modernité décrite par Mawdudi sous la direction de Hasan al-Banna. L'islamisme n'est pas déclin, retour à un passé historique idéalisé, comme nous l'avons déjà dit, il a l'idée d' «islamiser la modernité».

On a fait valoir que pour lutter contre l'injustice, l'inégalité, le détournement des traditions et la tromperie des pouvoirs injustes, il faudrait un retour à la pensée islamique, aux textes sacrés, à la charia et on ne devrait pas autorisé à imposer des modèles culturels, sociaux et politiques impériaux. Les résultats de la modernité doivent être considérés comme un développement technique dans chaque domaine, mais certains estiment que la société doit être protégée de l'influence héréditaire de l'Occident, qui se transforme en une nouvelle Jahiliyya. Ceci, bien sûr, concerne la vie religieuse, la vie sociale et la politique.

Dans ce cas, nous trouvons une formule politique dans la littérature politique des islamistes qui peut être perçue comme une synthèse sincère entre le double discours et les différentes traditions historiques, les formes acceptables démocratiques, entravées entre rejet définitif et réconciliation. 17

\section{Évaluation Générale}

En plus de toutes ces critiques et des systèmes alternatifs développés ci-dessus, les suggestions des penseurs occidentaux sont bien sûr également rencontrées. Selon Bellah, le plus important des problèmes de modernisation ne concerne peut-être pas l'Islam lui-même. L'Islam peut contribuer à la modernisation politique, familiale et personnelle, répondant efficacement aux besoins religieux de la société islamique moderne.18 Ernest Gellner souligne que bien que l'Islam ne soit pas une source de modernité, il peut être associé à ceux des rangs contemporaine. Les sociétés musulmanes peuvent se débarrasser de cette bifurcation. Ils n'ont pas à choisir entre Mahomet ou Marx. Psychologiquement le mélange semble être extrêmement pratique. La prémisse était que l'Islam ne serait pas compatible avec la modernité ou avec les exigences de la société industrielle.19

Comme Muhammad Arkoun le dit, dans le monde contemporain de l'Islam, il n'y a toujours pas un désir persistant de penser à résoudre la «vérité», une lutte persistante pour approfondir, une stricte harmonie interne et le respect des intérêts de la société et le droit de savoir, comme la pensée classique. Les musulmans ont tendance à s’impliquer empiriquement sous toutes les formes, mais ils ne peuvent exprimer leur riche expérience historique que dans un langage fragmenté et intermittent, soit islamique classique, soit idéologiquement quelque peu défectueux.

Enfin, le modernisme et l'Islam sont considérés comme incompatibles, mais cela ne signifie pas que le contraire de l'Islam est le modernisme. L'Islam n'est pas non plus perçu seulement

17 Jean Audibert, Jamais Je n'ai Cessé d'Apprendre l'Afrique, (Paris: Editions Karthala, 2006), p.191-192.

18 Robert N. Bellah, "İslamî Gelenek ve Modernleşme Problemleri”, Toplum Bilimleri Dergisi, 6/12, (2012), 247.

19 Ernest Gellner, Müslüman Toplum, (İstanbul: Kabalcı Yayınevi, 2012), p. 18, 130-131. 
comme un système spirituel. Conscient du potentiel de l'Islam, l'occidental a tenté d'orienter les musulmans et de modérer l'Islam. Par conséquent, il faut être prudents quant aux idées et méthodes innovantes. Les penseurs islamiques contemporains ont tendance à réduire la religion à l'idéologie. La réduction de l'Islam à l'idéologie et l'isolement et la politisation de ses dimensions idéologiques et spirituelles sont dangereuses. Sans comprendre les profondes ruptures épistémologiques qui requièrent de nombreuses innovations dans les domaines de la connaissance, notamment en sciences sociales, qui ont marqué la montée des temps modernes en Occident, les islamistes ont radicalement cherché à se détacher des bases philosophiques qui les engendrent et se tournent vers les sources. Il semble incohérent pour l'Occident d'adorer d'une part la science et la technologie et de l'autre, être contre les valeurs de la modernité. Cette réaction explique l'inadéquation des moyens conceptuels pour penser et dominer la complexité du processus qui résulte d'une longue dynamique historique en Occident. Ce n'est qu'en transcendant à d'autres sphères culturelles que l'ensemble des relations sociales et les valeurs peuvent être perturbées et que de nouveaux paradigmes rompus avec le passé peuvent se rejoindre.

C'est une confrontation que nous vivons aujourd'hui entre les valeurs traditionnelles auxquelles la société islamique est fondamentalement liée et la modernité importée, imposée et externe. Bien que la modernité ne soit pas totalement rejetée par les islamistes en tant que concept, on pense que la modernité devrait être produite pour elle-même, produite selon des critères internes et dans le respect des traditions.

En effet, la justice est produite en soi, car la modernité ne peut jamais être construite dans la mémoire des autres comme elle ne peut pas être liée à l'histoire des autres. Être musulman dans l'esprit de beaucoup aujourd'hui et s'exprimer en tant que musulman et être moderne, c'est abandonner son statut personnel.

Il convient de se demander si la relativité des penseurs islamiques contemporains à la tradition et à la proximité de ceux-ci avec le modernisme et les penseurs islamiques, qui s'opposent de la même manière au modernisme, critiquaient les paradigmes modernes. Aujourd'hui, on prétend que les discussions méthodologiques dans les sciences islamiques fondamentales sont basées sur le modernisme comme origine. En particulier, on peut mentionner sous quelles préoccupations sont formulés l'appel à revenir au Coran et l'ouverture de la porte de la jurisprudence. La relation révélation-raison, le problème de l'islamisation du savoir, la relation de la pensée mondiale et son approche à la science avec le modernisme peut être traité et on peut examiner si la pensée islamique contemporaine s'écarte des points originaux, quel genre de solutions elle affirme, si elle est affecté ou affectant.

Selon Burhan Ghalioun, «si le développement du nationalisme était le produit de la modernisation, l'islamisme contemporain est devenu le produit de leur échec ». "L'islamisme ne se développe que là où la faillite d'un nationalisme fort laisse un grand vide ». « L'islamisme s'est développé sur l'épave du nationalisme avant qu'il ne devienne son adjoint ».

Ils veulent réconcilier cette modernité au modèle local, simple et ultra matériel avec les valeurs de liberté, de justice et d'humanisme, considérées uniquement comme islamiques. Il y a une réaction à une modernité qui a été et est en effet appauvrie au nom des idéaux vu comme isla- 
miques et qui sont les valeurs et les idéaux de la modernité et de la sécularisation. Par conséquent, selon Burhan Ghalioun, l'islamisme n'est pas anti-modernité mais est à l'opposé de la modernité.

La ré-identification passe essentiellement par une réapparition du Coran et de la Sunna dans la vie quotidienne des sociétés musulmanes, et par le renforcement des musulmans leur propre histoire et leur passé; ce n'est pas un passé qui ne devrait jamais finir en soi, mais une transition nécessaire pour comprendre le présent par la science et la technologie.20

Comme la pensée islamique moderne a une infrastructure épistémologique enracinée, elle a le potentiel de jouer un rôle constructif et réparateur face aux problèmes modernes tels que le nationalisme moderne, la démocratie, l'État-nation, la modernité et le colonialisme. Si nous voulons dire ces choses brièvement et de manière concise; soutenir l'idée d'un monde islamique qui est le critère idéal pour tous d'abord et ensuite régler sérieusement les gigantesques changements sociaux et économiques qui ont émergé dans l'histoire de la pensée occidentale dans le contexte de leurs influences politiques et intellectuelles sur le monde islamique et finalement sur les sociétés contemporaines et le monde islamique, il est nécessaire de rendre normatif le système éducatif en termes de notre tradition et nos activités de production nationale.

\section{Références}

Adams, Charles J., "Fazlur Rahman ve Klasik Modernizm”, İslam ve Modernizm: Fazlur Rahman Tecrübesi Sempozyum Kitabı, İstanbul: İ. B. B. Yayınları, (1997).

Altun, Fahrettin, Modernleşme Kuramı, İstanbul: Küre Yayınları, 2011.

Arlantürk, Zeki-Akyüz, Niyazi, Din Sosyolojisi II, İstanbul: Çamlıca Yayınları, 2015.

Audibert, Jean, Jamais Je n'ai Cessé d'Apprendre l'Afrique, Paris: Editions Karthala, 2006.

Baykan, Erdal, "Modernliği ve Dindarlığı Uyuşum ve Ayrışma Hâsılasının Ötesinde Okumak Mümkün mü?”, Dindarlık Olgusu Sempozyum Tebliğ ve Müzakereleri, İstanbul: Kur’an Araştırmaları Vakfi, (2004).

Bellah, Robert N., “İslamî Gelenek ve Modernleşme Problemleri”, Toplum Bilimleri Dergisi, V. 6, n. 12, (2012).

Ben Elmostafa, Okasha, Les Mouvements Islamiques au Maroc, Paris: L'Harmattan, 2007.

Er, Neslihan, Moderniteyi İslamlaştırma Projesi: Al Adl Wal Ihsanne Cemaati Modeli, M.Ü. Sosyal Bilimler Enstitüsü, Yayınlanmamı̧̧ Y. Lisans Tezi, (2014).

Eren, Recep, "Prof. Dr. Mehmet S. Aydın’a göre Türk Toplumunun Modernleşmesi”, İstikamet, N. 3, (2011).

Gellner, Ernest, Müslüman Toplum, İstanbul: Kabalcı Yayınevi, 2012.

Küçükcan, Talip, "Modernleşme ve Sekülerleşme Kuramları Bağlamında Din, Toplumsal Değişme ve İslâm Dünyası", İslâm Araştırmaları Dergisi, ISAM, n. 13, (2005).

Lapidus, Ira M., Modernizme Geçiş Sürecinde İslam Dünyası, İstanbul: IFAV, 1996.

Şeriati, Ali, Medeniyet ve Modernizm, İstanbul: Yeni Zamanlar Yayınları, 2003.

Shayegan, Daryush, Yaralı Bilinç, İstanbul: Metis Yayınları, 2012.

Voll, John Obert, "Batı'nın Modernite ile Özdeşleştirilmesinin Yanlışlı̆̆ı”, Bilimname, n. 3, (2003).

20 İbrahim M. Abu-Rabi, Modernlik ve Çă̆daş İslam Düşüncesi, (İstanbul: Yöneliş Yayınları, 2003), p. 188. 